\title{
Non-Neurogenic Neurogenic Bladder
}

National Cancer Institute

\section{Source}

National Cancer Institute. Non-Neurogenic Neurogenic Bladder. NCI Thesaurus. Code C123206.

Bladder sphincter dysfunction in an individual with normal bladder innervation, which may lead to renal impairment. 